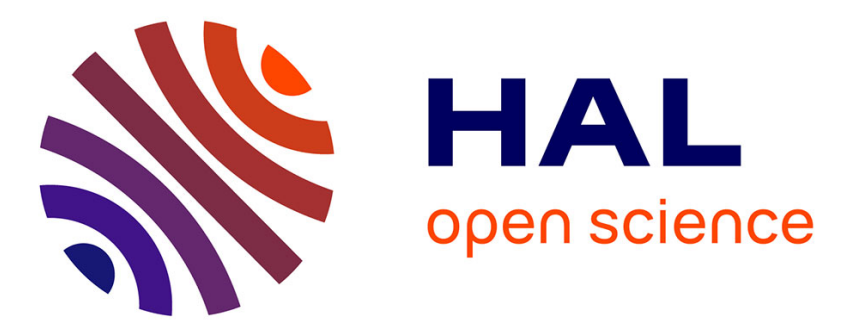

\title{
Scale analysis of the flame front in premixed combustion using Proper Orthogonal Decomposition
}

\author{
G. Maurice, F. Thiesset, F. Halter, Nicolas Mazellier, C. Chauveau, I. Gökalp,
} Azeddine Kourta

\section{- To cite this version:}

G. Maurice, F. Thiesset, F. Halter, Nicolas Mazellier, C. Chauveau, et al.. Scale analysis of the flame front in premixed combustion using Proper Orthogonal Decomposition. Experimental Thermal and Fluid Science, 2016, 73, pp.109 - 114. 10.1016/j.expthermflusci.2015.09.030 . hal-01660271

\section{HAL Id: hal-01660271 https://hal.science/hal-01660271}

Submitted on 28 Mar 2019

HAL is a multi-disciplinary open access archive for the deposit and dissemination of scientific research documents, whether they are published or not. The documents may come from teaching and research institutions in France or abroad, or from public or private research centers.
L'archive ouverte pluridisciplinaire HAL, est destinée au dépôt et à la diffusion de documents scientifiques de niveau recherche, publiés ou non, émanant des établissements d'enseignement et de recherche français ou étrangers, des laboratoires publics ou privés. 


\title{
Scale analysis of the flame front in premixed combustion using Proper Orthogonal Decomposition
}

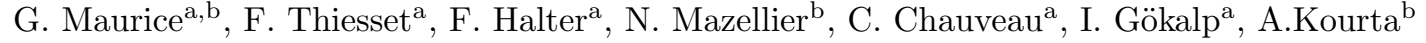 \\ ${ }^{a}$ CNRS ICARE, Avenue de la Recherche Scientifique, 45072 Orléans Cedex 2 France \\ ${ }^{b}$ University of Orléans, PRISME, EA 4229, 45072 Orléans, France
}

\begin{abstract}
The main objective of the present study is to explore the degree of interactions between turbulence and the flame front in the context of lean premixed combustion. Premixed methane Bunsen flames are investigated using Mie scattering at three different pressure magnitudes $(0.1,0.2$, and $0.3 \mathrm{MPa})$ and equivalence ratios $(0.6,0.7,0.8)$ through six operating conditions at the same mean bulk velocity $(3.6 \mathrm{~m} / \mathrm{s})$. Nearly homogeneous and isotropic turbulence is generated by a multi-scale grid consisting of three distinct perforated plates. The multi-scale nature of the flame front is characterized using an original method based on Proper Orthogonal Decomposition (POD). Particular emphasis is shed to the scales responsible for the flame brush and the wrinkling of the flame front. The flame front curvilinear length is analysed as a function of the mode (or equivalently the scale) and yields the well known Richardson plot that is usually observed using fractal analysis. The inner and outer cut-off length-scales, together with the fractal dimension can thus be evaluated using the present method. An analytical expression for unambiguously assessing the latter three parameters is introduced and compares favourably well with experimental data. Second, the reconstruction of the flame using only the first few modes suggests that, the flame brush is characteristic of a rather large-scale phenomena whilst the smallest scales act in decreasing the total flame brush.
\end{abstract}

Keywords: Premixed Turbulent Combustion, Flame Brush, Flame wrinkling

\section{Introduction}

Turbulence gives rise to a large and continuous range of scales. The largest eddies reflect the way kinetic energy is injected in the system and therefore depend on the type of flow. These large-scales are sometimes referred to as coherent structures whose topology and dynamic are strongly affected by initial and boundary conditions. In contrast, one frequently asserts that the anisotropic and nonuniversal influence of the largest scales diminishes during the first non-linear local interactions and is thus expected to decline at the smallest scales. Consequently, it is still often postulated that the smallest scales have the best prospect of being universal or quasi-universal [1]. Since, in premixed combustion, the corrugation of the flame front results mainly from the interaction with turbulence, it may be argued that such a statement holds also for the statistics of the flame front wrinkles at a given scale. This intuitive conjecture encourages us in exploring the multi-scale nature of turbulent

*Corresponding author: fabien.halter@cnrs-orleans.fr 
flames with the far aim of quantifying the degree of dependence of the large-scales wrinkles to initial and boundary conditions and assessing the degree of universality of the flame front at small-scales.

In the past-few years, the multi-scale facets of the flame front have been first analyzed using a fractal approach. More particularly, the scales distribution of the flame front has been generally inferred by means of the box-counting technique (see among others [2] and references therein). This approach led to the identification of the three distinct ranges in the wrinkling scales distribution. At large-scales, the production of wrinkles is almost negligible. As we travel through smaller scales, one may reach the outer length scale where the flame front starts being corrugated. Then, at intermediate scales, a power-law dependence of the wrinkling to the scale is observed with a scaling exponent related to the so-called fractal dimension. Finally, the smallest-scales, i.e. scales smaller than an inner cut-off length-scale are not efficient enough for corrugating the flame front, since viscous, kinematic and/or thermo-diffusive effects are dominant and preclude the creation of smaller wrinkles.

As in the field of turbulence, spectra have also been used for evaluating the distribution of scales of the flame front, leading to very similar deductions [3]. Note however that in practice, the flame front is not described by single valued spatial coordinates which makes the calculation of spectra rather tenuous, if not impossible. With this limitation in mind, it was noted however that the spectral exponent in the inertial range was close to previous estimations of the fractal dimension [3].

In the present study, we propose to explore the multi-scale facets of the flame front by means of Proper Orthogonal Decomposition (hereafter abbreviated by POD). POD belongs to a family of modal decomposition methods and is widely used in the field of fluid mechanics. Generally, use is made of POD to educe coherent structures from the randomly fluctuating flow. It has been introduced in fluid mechanics by Lumley [4] and the snapshot method which is more convenient for large data sets has been developed by Sirovich [5]. At this stage, it has to be emphasized that, unlike spectral or fractal approaches, POD has the tremendous advantage of allowing to finely reconstruct the instantaneous flame front mode-by-mode or equivalently scale-by-scale, and the contribution of each scale to the instantaneous flame brush or wrinkling can be inferred. Note that POD is a data driven technique meaning that the decomposition is based on a a priori basis. This implies that the relationship between a POD mode and a physical scale is not straightforward.

In the field of combustion, very few studies have focused on modal decompositions. It has been early used by Danby et al. [6] to investigate the auto ignition of inhomogeneous hydrogen air mixture. These authors tried several preprocessing techniques on the data-set in order to optimize the number of modes needed to minimize the reconstruction error. Later, using PIV data sets and OH-PLIF together with the extended POD methods [7] introduced by Borée [8], Duwig et al. used POD to study (i) thermo acoustics instabilities [9], (ii) the interaction of precessing vortex core with a swirling flame [10] and (iii) unsteady flames driven by acoustic perturbation. Finally, to characterize the flame shedding process behind a bluff-body [11], Kostka et al. used POD on flame chemiluminescence images to extract the coherent behavior and separate the energy at given mode into symmetric, asymmetric, and uncorrelated components.

Here, POD is applied to the flame front spatial coordinates extracted from Mie scattering tomography measurements. First, particular attention is paid to the scale distribution of wrinkles, by evaluating the curvilinear length of the flame front as a function of the scale. For this purpose, the correspondence between the mode number and a typical scale is successfully inferred. An analytical expression for assessing the inner and outer length-scale together with the scaling exponent is also proposed. Finally, the focus is rather on the large-scales and their contribution to the flame brush. 
The paper is organized as follows. First we describe the experimental set-up and measuring technique. Secondly, the POD algorithm as applied to the present configuration is outlined. Then, results for the curvilinear length and flame brush are presented and discussed. Findings are summarized in a final section.

\section{Experimental apparatus}

The turbulent methane/air premixed flame is generated via an axisymetric Bunsen burner with $D=25 \mathrm{~mm}$ in diameter. For all cases reported in this study, the bulk velocity $U_{d}$ of the fresh gases is $3.6 \mathrm{~m} / \mathrm{s}$. A turbulence generator located upstream from the burner exit enables to produce a nearly homogeneous and isotropic turbulence which has been fully detailed in Fragner et al. [12]. A schematic of the experimental set-up is given in Fig. 1. The burner is implemented in a pressure chamber that allows to regulate the pressure $P$ within the range 0.1 to $1 \mathrm{MPa}$. Experimental operating conditions are listed in Table 1. The instantaneous flame front is captured by means of laser tomography using a 15W $532 \mathrm{~nm}$ continuous laser light, the fresh gases being seeded with organic oil droplets. The flame front images are recorded with a phantom V1210 Phantom camera, using a $105 \mathrm{~mm}$ F2.8 lens, at a frame rate of $10000 \mathrm{fps}$ on a cropped sensor at $800 \times 384$ pixels. The physical field of view is $40.32 \mathrm{~mm}$ wide and $84 \mathrm{~mm}$ high which leads a spatial resolution of $0.10582 \mathrm{~mm} /$ pixel. For each experimental operating condition $N_{i m}=10000$ images were acquired. The instantaneous 2D flame front is binarised following a threshold algorithm. Firstly, a contrastlimited adaptive histogram equalization (CLAHE) is applied to the original images in order to optimize the contrast in the images. Then, to limit the pixelixation associated with the CLAHE, images are filtered using a gaussian filter of size equal to 4 times the spatial resolution. For the binarizing procedure, we use a standard treshold-based technique. More precisely, the histogram of the gray scale is calculated. The latter reveals two distinct peaks corresponding to the fresh and burned gas respectively. The treshold value for discrimating the flame contour is set as the average value between the gray scale of these two peaks. This binarization procedure leads irremediably to a digitization noise (pixelization) which, in the present case, is smoothed using a low-pass gaussian size equal to 3 times the spatial resolution. It was checked that doubling the size of the filter did not yield observable changes on the properties of interest (namely the flame wrinkling distribution). This indicates that the present measurements are well resolved and that the filter size that is used here is much smaller than that of the smallest flame wrinkling characteristic lengthscale. Then, the contour of the turbulent flame is extracted by means of an edge detection algorithm.

For the present study, we made the choice of focusing only on the longest contour representing the largest topologically connected object, whereas holes and pockets are not tacken into account. The contribution of these missing flame holes and pockets to e.g. the flame surface density was rather limited notwithstanding the relatively low turbulence intensity of our experiments. Future work is however needed to incorporate these disconnected objects into a more self-consistent description. 


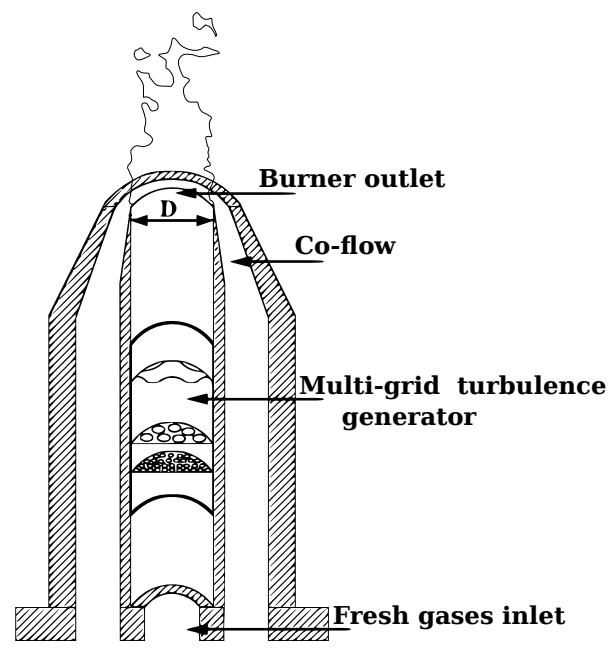

Figure 1: sketch of the turbulent bunsen premixed burner [12]

It is also important to mention that the oil boiling temperature is $250{ }^{\circ} \mathrm{C}$. Therefore, the flame fronts extracted by this tresholding algorithm correspond to isothermal surfaces in the flow.

Table 1: Operating conditions. $P$ is the pressure in the combustion chamber, $\phi$ is the equivalence ratio, $S_{l}$ is the laminar flame speed and $\delta_{l}$ is the laminar flame thickness.

\begin{tabular}{|c||cccc|}
\hline Case & $P(\mathrm{MPa})$ & $\phi$ & $S_{l}(\mathrm{~m} / \mathrm{s})$ & $\delta_{l}(\mathrm{~mm})$ \\
\hline \hline 1 & 0.1 & 0.6 & 0.113 & 0.197 \\
2 & 0.1 & 0.7 & 0.191 & 0.117 \\
3 & 0.1 & 0.8 & 0.226 & 0.083 \\
4 & 0.2 & 0.6 & 0.076 & 0.147 \\
5 & 0.3 & 0.6 & 0.058 & 0.127 \\
6 & 0.3 & 0.8 & 0.083 & 0.045 \\
\hline
\end{tabular}

\section{POD Algorithm}

The spatial coordinates $\left(x_{i, j}\right.$ and $\left.y_{i, j}\right)$ of the longest contour (the black curve in Fig. 2(a)) are re-sampled on a fixed size grid ( $N_{p}=2000$ sampling points) and rearranged in column vectors to combine them in a matrix called the snapshot matrix $X_{i j}$, with $i$ is the contour point number and $j$ is the time step number, viz. 


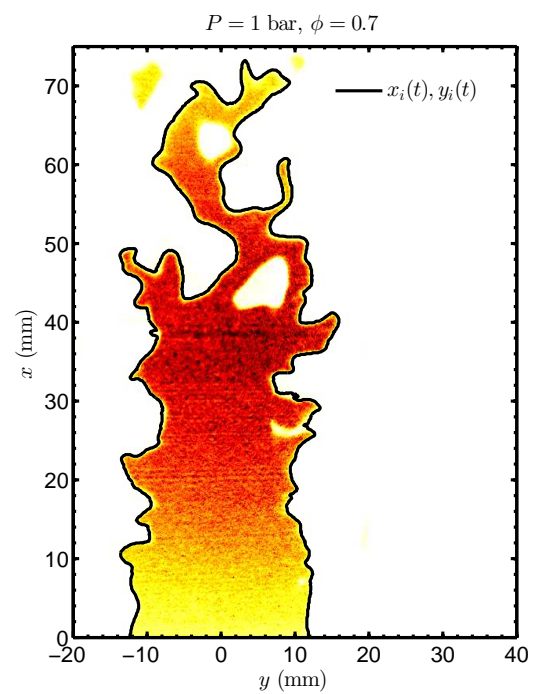

(a)

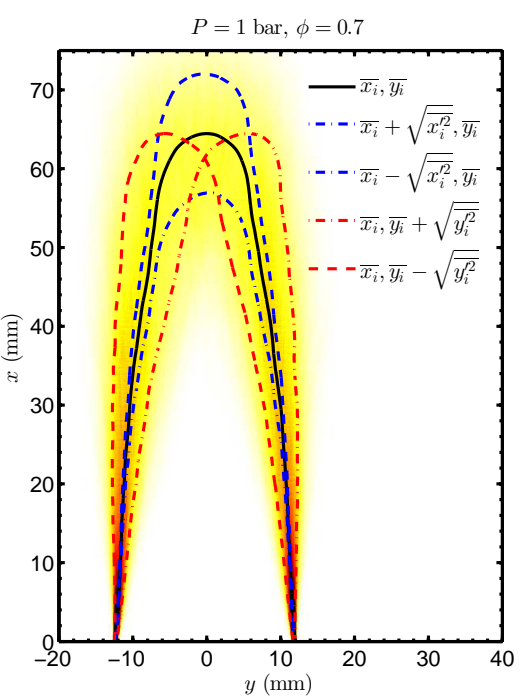

(b)

Figure 2: (a) Example of contour determination for case 2. (b) Flame position probability.

$$
X_{i, j}=\left(\begin{array}{ccccc}
x_{1,1} & \cdots & x_{1, j} & \cdots & x_{1, N_{i m}} \\
\vdots & \vdots & \vdots & \vdots & \vdots \\
x_{i, 1} & \cdots & x_{i, j} & \cdots & x_{i, N_{i m}} \\
\vdots & \vdots & \vdots & \vdots & \vdots \\
x_{N_{p}, 1} & \cdots & x_{N_{p}, j} & \cdots & x_{N_{p}, N_{i m}} \\
y_{1,1} & \cdots & y_{1, j} & \cdots & y_{1, N_{i m}} \\
\vdots & \vdots & \vdots & \vdots & \vdots \\
y_{i, 1} & \cdots & y_{i, j} & \cdots & y_{i, N_{i m}} \\
\vdots & \vdots & \vdots & \vdots & \vdots \\
y_{N_{p}, 1} & \cdots & y_{N, j} & \cdots & y_{N_{p}, N_{i m}}
\end{array}\right)
$$

From these snapshot matrix, one can compute the mean flame position $\overline{X_{i}}$, the standard deviation of the flame positions $\sqrt{\overline{X_{i}^{\prime 2}}}$, the flame position probability (Fig. 2(b)) and the normalized flame position matrix

$$
\chi_{i, j}=\frac{X_{i, j}-\overline{X_{i}}}{\sqrt{\overline{X_{i}^{\prime 2}}}}
$$

The POD algorithm is applied to the normalized snapshot matrix $\chi_{i, j}$. The algorithm consists in determining a decomposition of the snapshot matrix in a sum of deterministic functions $\Phi_{i, k}$ 

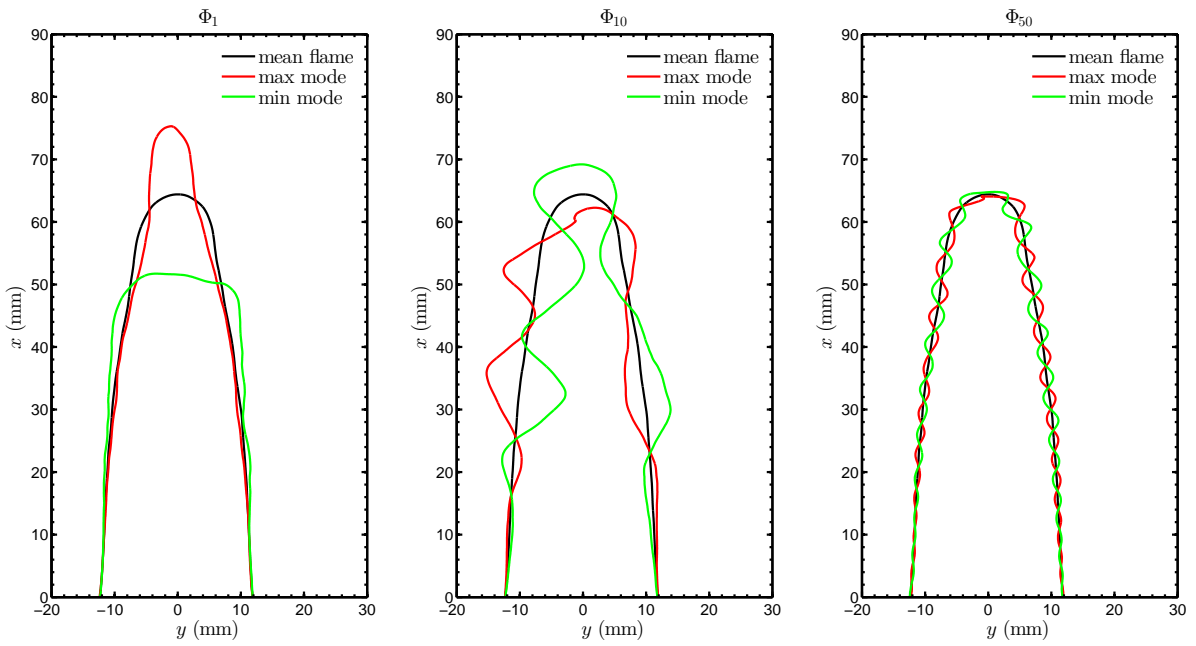

Figure 3: Mode 1, 10 and 50 superposed on the mean flame contour for case 2

called modes, multiplied by temporal coefficients $\psi_{k}(t)$ or $\psi_{k, j}$ and a weight $p_{k}$.

$$
\chi_{i, j}=\sum_{k=1}^{N_{m}} \Phi_{i, k} w_{k} \Psi_{k, j}
$$

The mathematical constraint on the deterministic functions and temporal coefficients is that they are both orthogonal regarding the inner product on the integrable squared function space $\mathcal{L}^{2}$.

$$
\begin{aligned}
& \sum_{j} \Psi_{k, j} \Psi_{l, j}=\delta_{k, l} \\
& \sum_{j} \Phi_{j, k} \Phi_{j, l}=\delta_{k, l}
\end{aligned}
$$

By rewriting equation 3 in a matrix formulation

$$
\chi=\Phi \times W \times \Psi
$$

and using the mathematical constraints described in equations 4 and 5 , one can readily show that the temporal correlation matrix $R$ can be written as:

$$
R=\chi^{T} \chi=\Psi^{T} W^{2} \Psi=\Psi^{-1} \Lambda \Psi
$$

Since the matrix $\Psi$ is orthogonal, one can identify from equation 7 the eigen value decomposition of the matrix $R$ where $W^{2}=\Lambda$ is the diagonal eigen value matrix and $\Psi$ is the eigen vector matrix. The POD modes $\Phi_{i}$ are obtained by computing the projection of the snapshots on the eigen vectors. The eigen values $\lambda_{i}$ respectively represent the energy associated to the variation of modes $\Phi_{i}$ and by convention the modes are sorted from the highest to the smallest eigen value. The detailed mathematical description of POD analysis and many applications can be found in the review by 

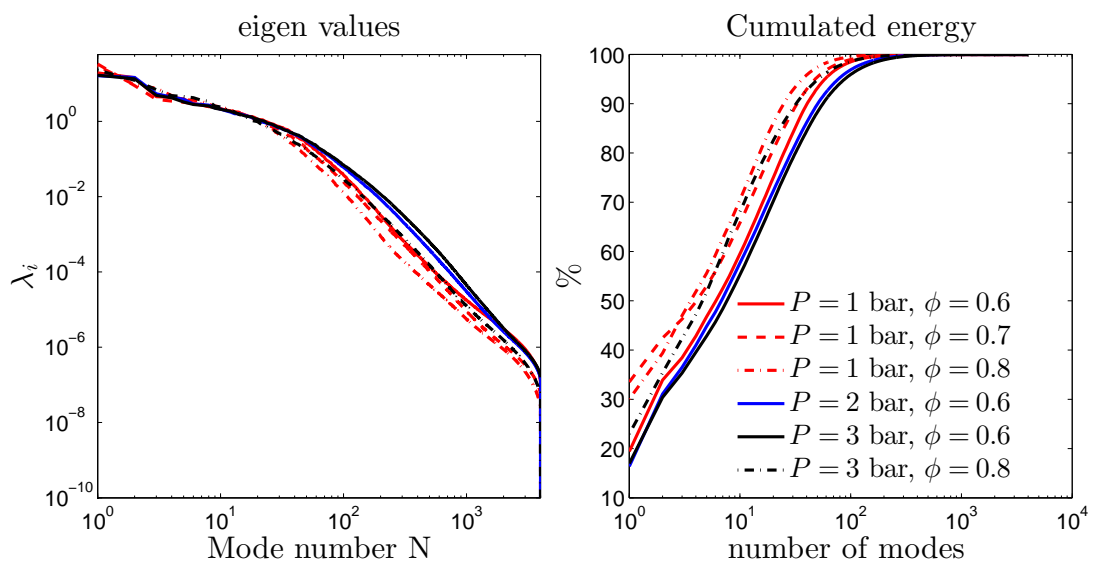

Figure 4: Eigen Value spectra and cumulative energy as a function of the mode number

Berkooz [13]. Before using POD algorithm on the normalized flame positions, the algorithm has been assessed on a benchmark data-set. Each experimental case has been explored using the POD algorithm which provides $N_{p}$ modes sorted by decreasing energy. The first modes correspond to large scale motions which are mostly perceptible on the top of the flame, whilst higher order modes correspond to small scale oscillations (See Fig. 3). The eigen value $\lambda_{i}$ spectra of the decomposition are plotted in Fig. 4. They represent the relative energy associated with each mode and their contribution to the total variance. A careful analysis of Fig. 4 reveals that the effect of an increase of pressure is to reduce the energy decay of high-order modes. In other words, this shows that the small-scale flame wrinkling is enhanced as pressure increases, as expected from the enhancement of the small-scales activity $[14,15]$. Moreover as the equivalence ratio increases the energy decay of high order modes is accentuated. Since the laminar flame speed increases with equivalence ratio, the small scales disappears faster in agreement with the suggestion of Peters [16]. At this point it is also important to mention that since the POD algorithm is performed on the flame contours, the obtained eigen value spectra directly correspond to the relative contribution of each spatial patterns (i.e., POD modes) to the flame brush

\section{Decomposition of the curvilinear flame length}

In order to further assess contribution of each mode to the flame front statistics, we first introduce the mean flame length $L$ recovered with $n$ modes.

$$
L(n)=\sqrt{\int_{0}^{N_{p}} \sqrt{\left(\frac{\partial x_{n}(\tau, t)}{\partial \tau}\right)^{2}+\left(\frac{\partial y_{n}(\tau, t)}{\partial \tau}\right)^{2}} d \tau}
$$

where $\tau$ is the curvilinear parameter. To further proceed, we now have to relate a specific scale $r$ to a mode number $n$. For this purpose, a spatial Fourier transform is applied to each mode and the scale $r(n)$ is associated with the wavelength of maximum spectral energy. These maxima (red curve in Fig. 5(a)) has been smoothed using an auto-regressive average (black line). Fig. 5(a) shows that the evolution of the domininant wavelength is quasi linear for $n<10^{3}$. For modes $n$ larger 


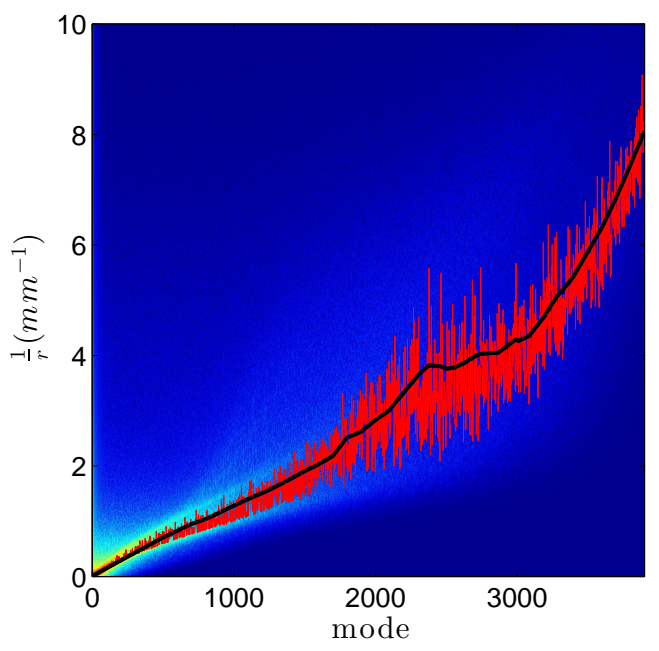

(a)

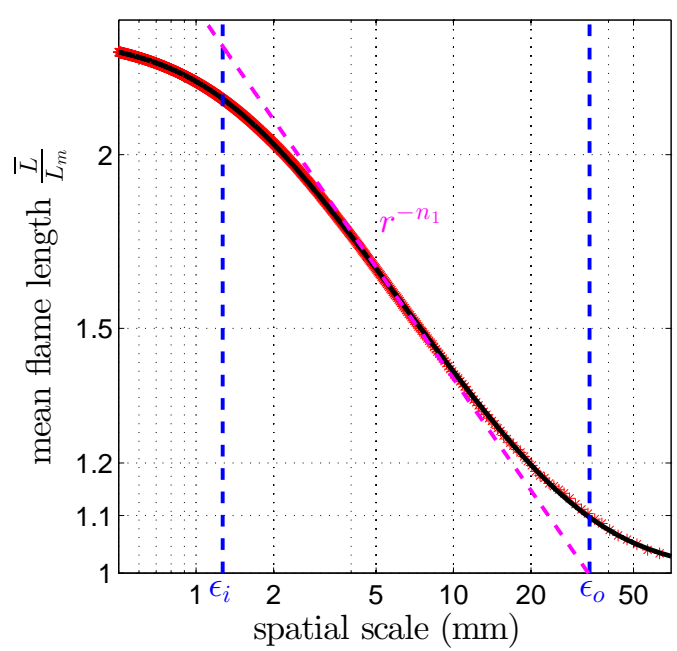

(b)

Figure 5: (a) Spatial Fourier transform of the POD modes for flame 2. (b) Example of a fitted flame length evolution for flame 5 .

than $10^{3}$, the correspondence between $n$ and $r$ starts being contaminated by noise since there is no further energy in the signal (see Fig. 4).

The curvilinear flame length $L(r)$ normalized by the length of the mean flame contour $L_{m}$ for Case 5 is presented in Fig. 5(b). Noticeable is the (inverse) S-shape of this curve, as generally observed using a fractal approach and the box-counting technique[2]. One observes three distinct regimes. At large scales $(r>35 \mathrm{~mm})$, the flame curvilinear length tends to that of the average flame front $L_{m}$. Then $L(r)$ sharply increases as $r$ decreases before a sudden change in behaviour at even smaller scales $r<1 \mathrm{~mm}$ for which $L(r)$ is likely to reach a constant value. In order to provide an accurate and unambiguous estimation for the outer length-scale $\epsilon_{o}$, the inner cut-off $\epsilon_{i}$ and the scaling exponents $n_{1}$, the evolution of $L(r)$ is fitted using an expression similar to that proposed by Batchelor [17]

$$
\frac{L(r)}{L_{m}}=\left(\frac{\epsilon_{o}}{\epsilon_{i}}\right)^{n_{1}}\left[1+\left(\frac{r}{\epsilon_{o}}\right)^{2}\right]^{\frac{n_{1}}{2}}\left[1+\left(\frac{r}{\epsilon_{i}}\right)^{2}\right]^{-\frac{n_{1}}{2}} .
$$

As illustrated in Fig. 5(b), this expression is perfectly suited to describe the evolution of $L$ over the entire range of $r$. Therefore, using this expression for $L(r)$, the three parameters $\epsilon_{o}, \epsilon_{i}$ and $n_{1}$ are computed directly using a non linear least-square fit and are not determined by subjectively optimizing the extent of the plateau in the compensated inertial range.

All experimental conditions have been analysed with this method. Values for $\epsilon_{i}, \epsilon_{o}$ and $n_{1}$ are summarized in Table 2. The outer-length scale $\epsilon_{o}$ appears to be sightly smaller (e.g., 20-40 mm) than the flame height $H_{f}$ and significantly larger than the integral length-scale $(\approx 5 \mathrm{~mm})$. Arguably, $\epsilon_{o}$ is likely to be related to the large-scale fluctuations observed on the top of the flame (see Fig. 3). As far as the inner scale is concerned, one notices that $\epsilon_{i}$ significantly decrease when the pressure in 
Table 2: Mean flame length scaling parameters

\begin{tabular}{|c||cccc|}
\hline Case & $\epsilon_{o}(\mathrm{~mm})$ & $\epsilon_{i}(\mathrm{~mm})$ & $H_{f}(\mathrm{~mm})$ & $n_{1}$ \\
\hline \hline 1 & 28 & 5.25 & 66.6 & 0.222 \\
2 & 39 & 4.64 & 44.12 & 0.223 \\
3 & 34 & 3.8 & 30.7 & 0.24 \\
4 & 40 & 2.01 & 57.03 & 0.239 \\
5 & 34 & 1.36 & 55.02 & 0.269 \\
6 & 28 & 0.95 & 21.16 & 0.269 \\
\hline
\end{tabular}

the chamber is increased. This is in perfect agreement with the deductions drawn by Kobayashi et al. $[14,15]$, emphasizing that the evolution of $\epsilon_{i}$ with pressure follows from the enhancement in the small scale activity associated with a decrease of the kinematic viscosity. The scaling exponent $n_{1}$ which is related to the fractal dimension $D_{f}$ by $D_{f}=2+n_{1}$ is also consistent with the assessments of Smallwood et al. [2].

Altogether, present results show that POD is perfectly suited for analysing the multi-scale nature of the flame front and recovers previous findings using fractal approaches

\section{Decomposition of the flame brush}

We now turn our attention to the contribution of each scale to the flame brush. It is usual to relate the flame brush to the RMS of the probability density function of finding the flame front at a particular location $x, y$ (see e.g. [3] among other). As mentioned earlier, the outer and inner scales are the bounding scales of the flame wrinkling phenomenon. With the aim of assessing the contribution of the large and small scales to the flame brush, the POD technique allows us to reconstruct the flame evolution from the first mode to the mode corresponding to the outer scale $\epsilon_{o}$ and from the latter to the mode corresponding to the inner scale $\epsilon_{i}$. Results are plotted in Fig. 6 and compared to the whole flame position probability. Fig. 6 shows that the flame brush is characteristic of a rather large-scale phenomena since the probability of finding the flame front at a given position is well represented by the first few modes. Note however that the flame brush reconstructed with the first modes is slightly larger than that reconstructed using all modes. This indicates that the contribution of each scale to the flame brush is not an additive process and that, on the contrary, smaller scales act in diminishing the flame brush.

\section{Summary}

The multi-scale facets of turbulent Bunsen flame front are investigated using Mie scattering images for pressure levels varying from 0.1 to $0.3 \mathrm{MPa}$ and equivalence ratios from 0.6 to 0.8 . We propose an alternative method than spectral or fractal approaches by applying Proper Orthogonal Decomposition to the flame contours. The present study indicates that POD is perfectly suited for this task and that unlike previous techniques, POD has the tremendous advantage of allowing the instantaneous flame front to be reconstructed using a prescribed number of modes. However, the main difficulty when using POD is to relate a mode number to a physical length-scale. Here, a spatial Fourier transform of the flame contour positions at a given mode $n$ is performed and a linear dependence between $n$ and $r$ is emphasized. 

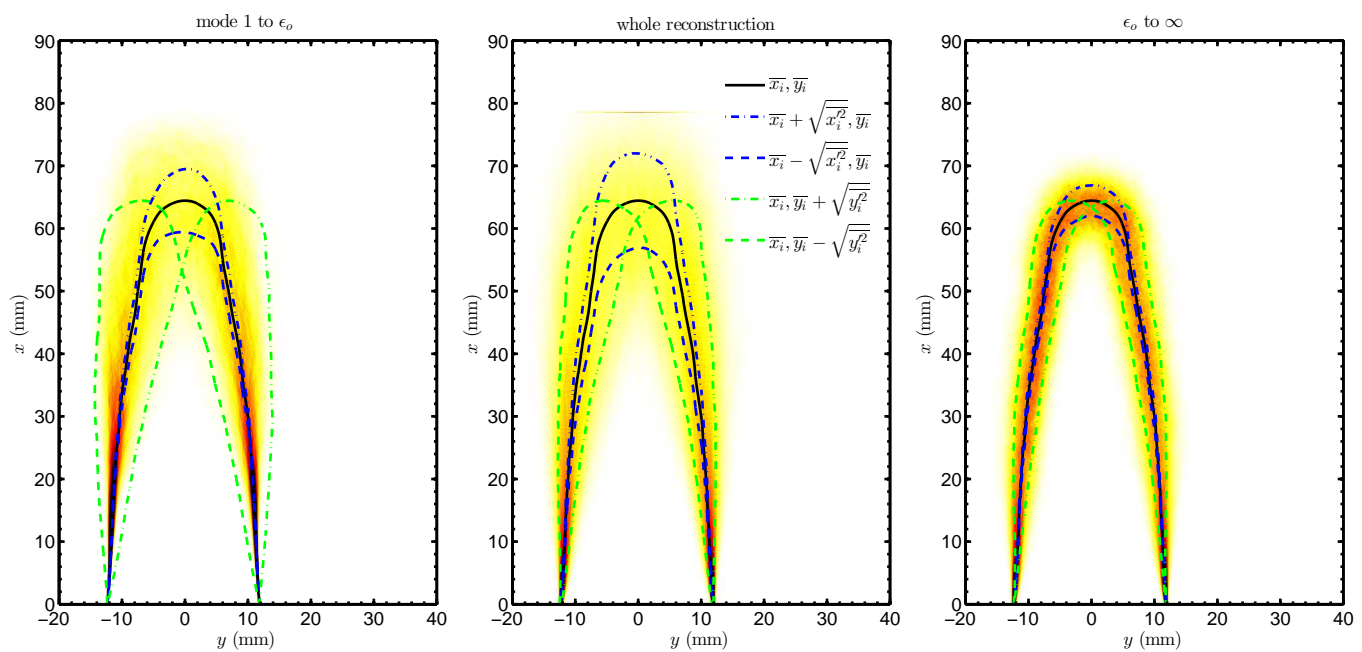

Figure 6: Flame position probability reconstructions for flame 2

Then, preliminary results for the curvilinear flame length and the flame brush are presented. Unlike previous efforts, a novel expression for the curvilinear length $L(r)$ which compares favorably well with experimental data is proposed to unambiguously infer the inner and outer length-scales and the fractal dimension. The values and evolutions with pressure and equivalence ratio of $\epsilon_{o}, \epsilon_{i}$ and $n_{1}$ is consistent with previous findings $[2,14,15]$. Then, attention is paid to the flame brush estimated from the RMS of finding the flame front at a given spatial location $x, y$. It is thus shown that the flame brush is characteristic of a rather large scale phenomenon and that small scales act in decreasing the flame brush.

\section{Acknowledgement}

The authors whish to thanks the Agence National de la Recherche for the financial financial support under the project IDYLLE. The authors are also thankful to the CNRS, the University of Orléans, and the French Government Programme through the program "investissement d'avenir" LABEX CAPRYSSES.

\section{References}

[1] K. R. Sreenivasan, R. A. Antonia, The phenomenology of small-scale turbulence, Annu. Rev. Fluid Mech. 29 (1997) 435-472. doi:10.1146/annurev.fluid.29.1.435.

[2] G. J. Smallwood, O. L. Gülder, D. R. Snelling, B. M. Deschamps, I. Gökalp, Characterization of flame front surfaces in turbulent premixed methane/Air combustion, Combust. Flame 101 (4) (1995) 461-470. doi:http://dx.doi.org/10.1016/0010-2180(94)00226-I.

[3] S. Kheirkhah, O. L. Gülder, Turbulent premixed combustion in V-shaped flames: Characteristics of flame front, Phys. Fluids 25 (5) (2013) 055107. doi:10.1063/1.4807073. 
[4] J. L. Lumley, Computational modeling of turbulent flows, Advances in Applied Mechanics, Hermes sciences, 1978.

[5] L. Sirovich, M. Kirby, M. Winter, An eigenfunction approach to large scale transitional structures in jet flow, Phys. Fluids 2 (2) (1990) 127. doi:http://dx.doi.org/10.1063/1.857815.

[6] S. J. Danby, T. Echekki, Proper orthogonal decomposition analysis of autoignition simulation data of nonhomogeneous hydrogenair mixtures, Combust. Flame 144 (1-2) (2006) 126-138. doi:10.1016/j.combustflame.2005.06.014.

[7] C. Duwig, P. Ludiciani, Extended Proper Orthogonal Decomposition for Analysis of Unsteady Flames, Flow Turb. Combust. 84 (1) (2010) 25-47. doi:10.1007/s10494-009-9210-6.

[8] J. Borée, Extended proper orthogonal decomposition: a tool to analyse correlated events in turbulent flows, Exp. Fluids 35 (2) (2003) 188-192. doi:10.1007/s00348-003-0656-3.

[9] C. Duwig, C. Fureby, Large eddy simulation of unsteady lean stratified premixed combustion, Combust. Flame 151 (12) (2007) 85-103. doi:10.1016/j.combustflame.2007.04.004.

[10] C. Duwig, L. Fuchs, Large eddy simulation of vortex breakdown/flame interaction, Phys. Fluids 19 (7) (2007) 075103. doi:10.1063/1.2749812.

[11] S. Kostka, A. C. Lynch, B. C. Huelskamp, B. V. Kiel, J. R. Gord, S. Roy, Characterization of flame-shedding behavior behind a bluff-body using proper orthogonal decomposition, Combust. Flame 159 (9) (2012) 2872-2882. doi:10.1016/j.combustflame.2012.03.021.

[12] R. Fragner, N. Mazellier, F. Halter, C. Chauveau, I. Gökalp, Multi-scale high intensity turbulence generator applied to a high pressure turbulent burner, Flow Turb. Combust. 84 (1) (2014) 25-47. doi:10.1007/s10494-014-9556-2.

[13] G. Berkooz, P. Holmes, J. L. Lumley, The Proper Orthogonal Decomposition in the Analysis of Turbulent Flows, Annu. Rev. Fluid Mech. 25 (1993) 539-575. doi:10.1146/annurev.fl.25.010193.002543.

[14] H. Kobayashi, T. Kawahata, K. Seyama, T. Fujimari, J.-S. Kim, Relationship between the smallest scale of flame wrinkles and turbulence characteristics of high-pressure, hightemperature turbulent premixed flames, Proc. Combust. Inst. 29 (2) (2002) 1793-1800. doi:10.1016/S1540-7489(02)80217-6.

[15] H. Kobayashi, K. Seyama, H. Hagiwara, Y. Ogami, Burning velocity correlation of methane/air turbulent premixed flames at high pressure and high temperature, Proc. Combust. Inst. 30 (1) (2005) 827-834. doi:10.1016/j.proci.2004.08.098.

[16] N. Peters, Laminar flamelet concepts in turbulent combustion, Proc. Combust. Inst. 21 (1988) 1231-1250. doi:http://dx.doi.org/10.1016/S0082-0784(88)80355-2.

[17] G. K. Batchelor, Pressure fluctuations in isotropic turbulence, Proc. Camb. Phi. Soc. 47 (1951) 359-374. doi:10.1017/S0305004100026712. 